\title{
Thermal Inactivation Model for Hepatitis E Virus (HEV)
}

Area of research interest: Foodborne pathogens

Study duration: 2019-03-01

Project status: Completed

Project code: FS301062

Conducted by: Glasgow Caledonian University

Date published: 19 January 2022

DOI: https://doi.org/10.46756/sci.fsa.sdt366

\section{Background}

Hepatitis $E$ is an emerging issue, with the number of confirmed cases in the UK increasing in 2009-2015, and decreasing slightly in 2016 and 2017. There is some epidemiological evidence of an association of this virus with undercooked pork and pork products. Currently, there is no standardized method for evaluating thermal stability of HEV and also a lack of a suitable assay that can distinguish between intact HEV that can cause an infection and damaged virus which is not capable of causing an infection. This has raised concerns as it is extremely difficult to extrapolate the risk from pork products in relation to cooking practices. We are seeking to address this knowledge gap, which will not only inform our risk assessment, but will also provide an indication if cooking is sufficient to inactivate the virus in foods.

\section{Objective and Approach}

The aim of this study is to develop a model which will efficiently predict thermal inactivation for HEV. In the Advisory Committee on the Microbiological Safety of Food (ACMSF) 2015 foodborne virus report, the 2016 jointly organised FSA-European Food Safety Authority (EFSA) foodborne virus workshop and the 2017 EFSA Scientific Opinion on HEV all concluded that there remain significant evidence gaps on HEV and identified research priorities including the need for further research on the heat stability and inactivation of HEV in foods.

A critical review of the peer-reviewed and grey literature relating to thermal inactivation of HEV in various food products will be carried out. Quantitative data on relevant parameters, such as time, temperature, matrix composition and virus source, will be recorded. Gaps in the published literature will be addressed by using data obtained from similar mammalian viral pathogens (surrogate studies), or through additional experimental work.

A mathematical model will be constructed from this quantitative data, which will predict HEV elimination through cooking regimes used by consumers and caterers in different food matrices. The model will be flexible in terms of HEV infectivity and it will allow the incorporation of additional data as new research becomes available. Validation of the model will be carried out using novel experimental data to ensure that it is fit-for-purpose and is quality assured.

The model developed in this research project can be used to inform the development of risk assessments, FSA advice to consumers and control measures in food safety management plans in industry, as well as identify where future surveillance and research activities are needed. 


\section{Results}

The study had 3 priorities, the outcomes of which are listed below:

- The study collected HEV thermal inactivation data from the published scientific literature. In total, 196 potentially suitable references were identified by the literature search, out of which 11 contained usable quantitative data. The data was inconsistent and considered insufficient for creating a model.

- There was a lack of data at the higher end of the temperature range, $70-95^{\circ} \mathrm{C}$, which is relevant to cooking. This indicated a gap in the information and affected the ability to assess the real impact of thermal inactivation on HEV. Therefore, extra data was generated from experiments that measured HEV RNA in saline solution before and after heating to 70 , $75,80,85,90$, and $95^{\circ} \mathrm{C}$.

- Data from the above steps was used to develop a model describing the effects of heating on HEV levels. A user-friendly model was generated, which can be populated with further thermal inactivation data, as it becomes available.

- The findings indicate that while traces of HEV can be identified at various temperatures, there is no reliable method currently to determine whether HEV particles are infectious or not.

The model can be accessed via the FSA's open data catalogue.

\section{Report}

PDF

View Thermal Inactivation Model for Hepatitis E Virus as PDF(Open in a new window) (4.96 MB) 